\title{
Chromospheric observations and magnetic configuration of a supergranular structure ${ }^{\star}$
}

\author{
Carolina Robustini, Sara Esteban Pozuelo, Jorrit Leenaarts, and Jaime de la Cruz Rodríguez
}

\author{
Institute for Solar Physics, Department of Astronomy, Stockholm University, AlbaNova University Centre, \\ 10691 Stockholm, Sweden \\ e-mail: carolina.robustini@astro.su.se
}

Received 17 April 2018 / Accepted 23 October 2018

\begin{abstract}
Context. Unipolar magnetic regions are often associated with supergranular cells. The chromosphere above these regions is regulated by the magnetic field, but the field structure is poorly known. In unipolar regions, the fibrillar arrangement does not always coincide with magnetic field lines, and polarimetric observations are needed to establish the chromospheric magnetic topology.

Aims. In an active region close to the limb, we observed a unipolar annular network of supergranular size. This supergranular structure harbours a radial distribution of the fibrils converging towards its centre. We aim to improve the description of this structure by determining the magnetic field configuration and the line-of-sight velocity distribution in both the photosphere and the chromosphere. Methods. We observed the supergranular structure at different heights by taking data in the Fe I 6301-6302 and the Ca II H\&K spectral lines with the CRisp Imaging SpectroPolarimeter (CRISP) and CHROMospheric Imaging Spectrometer (CHROMIS) at the Swedish 1-m Solar Telescope. We performed Milne-Eddington inversions of the spectropolarimetric data of $\mathrm{Fe}_{\text {I }} 6301-6302 \AA$ and applied the weak field approximation to Ca II $8542 \AA$ data to retrieve the magnetic field in the photosphere and chromosphere. We used photospheric magnetograms of CRISP, Hinode Solar Optical Telescope spectropolarimeter, and Helioseismic and Magnetic Imager to calculate the magnetic flux. We investigated the velocity distribution using the line-of-sight velocities computed from the Milne-Eddington inversion and from the Doppler shift of the $K_{3}$ feature in the Ca II $\mathrm{K}$ spectral line. To describe the typical spectral profiles characterising the chromosphere above the inner region of the supergranular structure, we performed a $K$-mean clustering of the spectra in Ca II $\mathrm{K}$.

Results. The photospheric magnetic flux shows that the supergranular boundary has an excess of positive polarity and the whole structure is not balanced. The magnetic field vector at chromospheric heights, retrieved by the weak field approximation, indicates that the field lines within the supergranular cell tend to point inwards, and might form a canopy above the unipolar region. In the centre of the supergranular cell hosting the unipolar region, we observe a persistent chromospheric brightening coinciding with a strong gradient in the line-of-sight velocity.
\end{abstract}

Key words. methods: observational - Sun: chromosphere - Sun: photosphere - Sun: magnetic fields

\section{Introduction}

Supergranulation is a poorly understood phenomenon of the solar atmosphere. Its origin is still under debate and several different models have been proposed. How the magnetic field and supergranulation are dynamically related remains unclear. However, it is well known that the velocity field in the supergranulation advects the vertical magnetic field to the boundaries of the supergranular cells where it creates a magnetic network (Tian et al. 2010; Ishikawa \& Tsuneta 2011; Orozco Suárez et al. 2012; Rincon et al. 2017).

One of the first models of the magnetic field above supergranular cells in the quiet Sun (QS) was proposed by Gabriel (1976). Assuming a potential field in a unipolar cell, he suggested that all the flux at the photosphere is concentrated on the supergranular boundaries while the inner cell is fieldfree. According to his model, the boundary field expands into the higher layers in a canopy shape and it becomes uniform above the cell when it reaches the corona. The study of Jones \& Giovanelli (1983) provided the first observational support to this model. They reported on six unipolar regions where the main

\footnotetext{
* The movie associated to Fig. 2 is available at https:// www . aanda.org
}

polarity field lines fan out towards the upper layers of the atmosphere. The extent of the unipolar regions usually coincides with supergranule boundaries. Further studies supported the presence of a low-lying magnetic canopy (e.g. Solanki \& Steiner 1990). However, there is no consensus about the presence of a field canopy above supergranular cells.

Alternatively, Schrijver \& Title (2003) proposed that a significant contribution to the photospheric flux is located in the inner supergranular cell. In such a scenario, a relatively strong internetwork can produce a low-lying canopy of field lines connecting the mixed polarities in the internetwork to the network boundaries, and this may translate into a low-lying canopy of fibrils (see also Aiouaz \& Rast 2006; Rutten 2006).

The arrangement of fibrils in the chromosphere is expected to mainly follow the magnetic field lines (de la Cruz Rodríguez \& Socas-Navarro 2011; Schad et al. 2013; Leenaarts et al. 2015; Martínez-Sykora et al. 2016; Zhu et al. 2016; Asensio Ramos et al. 2017), but this dependency becomes rather unclear in unipolar regions.

Reardon et al. (2011) observed a unipolar region of $\sim 40^{\prime \prime}$ diameter in the line core of Ca II $8542 \AA$, where all the fibrils crossing the unipolar region were completely confined within the cell interior, as also described in Foukal (1971). 
These fibrils appear to connect same polarity concentrations as they originate from the stronger field patches in the cell boundaries. However, Reardon et al. (2011) pointed out that usually only one footpoint is clearly rooted in the network, suggesting a rather complex magnetic topology above the unipolar region.

The parallel fibril arrangement observed by Reardon et al. (2011) is commonly known as chain, and it is one of the possible organised distributions into which fibrils are clustered (Tsiropoula et al. 1993). An alternative distribution is called rosette and it consists of a radial arrangement of fibrils at the vertices of the supergranular cells. At this location, the magnetic field is usually quite concentrated and can reach up to a few kilogausses. The strong magnetic field is thought to be the cause of the brightening observed in the rosette cores (Dara-Papamargaritis \& Koutchmy 1983). Tsiropoula et al. (1993) measured a blue-shift in the $\mathrm{H} \alpha$ spectral line core, at the very centre of the rosette and on the top of the radial fibril. They interpreted this as an upflow, while they also observed a downflow located at the fibril footpoints. Typical values for the rosette diameter ranges from $\sim 10^{\prime \prime}$ (Mooroogen et al. 2017) up to $\sim 50^{\prime \prime}$ (Tziotziou et al. 2003).

In this paper, we present high-resolution chromospheric observations of a unipolar region enclosed in a supergranular structure (SGS), with a radial arrangement of the fibrils. The novelty of these data lies in (1) narrow-band images in three different chromospheric spectral lines, including Ca II $\mathrm{H} \& \mathrm{~K}$ spectrally resolved observations with the new CHROMospheric Imaging Spectrometer (CHROMIS) at the Swedish 1-m Solar Telescope (SST, Scharmer et al. 2003), (2) and the peculiar morphology of the observed structure. The latter recalls of a chromospheric rosette, although the convergence point of the fibrils is located at the centre of the supergranular cell. Since the fibril distribution inside the unipolar cell cannot always be trusted as a magnetic field tracer, we aim to establish the magnetic field topology using polarimetry. We also aim to investigate the dynamics of the fibrils using line-of-sight (LOS) velocity information.

\section{Observations and data reduction}

On 2017 April 20, the observed unipolar region was located close to the active region NOAA 12651 on the east side of the $\operatorname{limb}\left(X=-845^{\prime \prime}, Y=261^{\prime \prime}\right)$. This target was observed between 09:41 and 10:01 UT with a heliocentric angle $\sim 68^{\circ}(\mu=0.37)$. Observations were carried out using the CRisp Imaging SpectroPolarimeter (CRISP, Scharmer 2006; Scharmer et al. 2008) and CHROMIS, at the SST. CRISP data were recorded along the following wavelength positions:

- Hi $6563(\mathrm{H} \alpha)$, at 25 equidistant positions between 6560.6 and $6565.4 \AA$ with $\Delta \lambda=200 \mathrm{~m} \AA$;

- Fe I 6301, at ten non-equidistant positions between 6300.7 and $6301.2 \AA$ with $\Delta \lambda=40 \mathrm{~m} \AA$, except for $\Delta \lambda=100 \mathrm{~m} \AA$ between the three positions in the blue wing and two positions in the red wing closest to the continuum;

- Fe I 6302, at 11 equidistant positions between 6301.8 and 6302.2 with $\Delta \lambda=40 \mathrm{~m} \AA$;

- Ca II 8542, at 17 positions between 8541.0 and $8543.0 \AA$ with $\Delta \lambda=100 \mathrm{~m} \AA$, except for $\Delta \lambda=200 \mathrm{~m} \AA$ between the two positions in the red and in the blue wings closer to the continuum.

The time cadence is $\sim 39 \mathrm{~s}$. The pixel size and the spectral resolution $R$ at $6302 \AA$ are $0 .{ }^{\prime \prime} 059$ and $R=\lambda / \delta \lambda \approx 1.14 \times 10^{5}$,

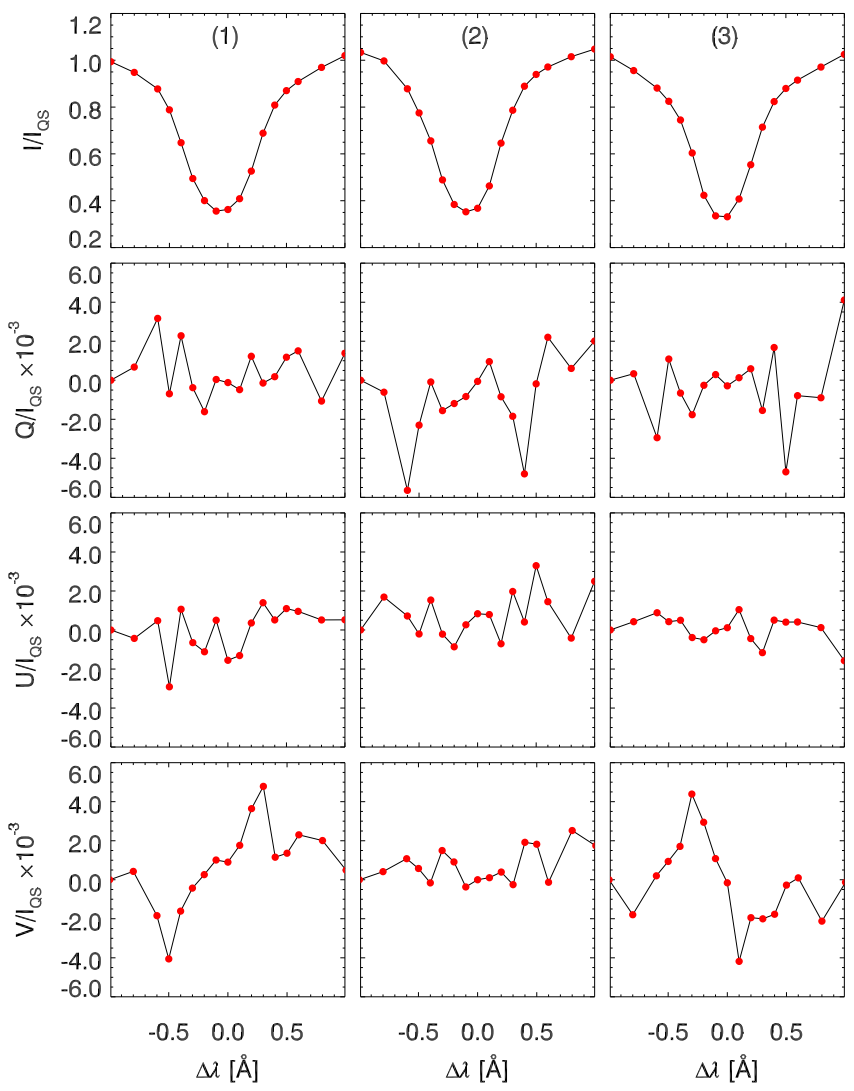

Fig. 1. Typical Stokes profiles of Ca II $8542 \AA$ observed within the SGS and corresponding to pixels $1,2,3$ in Fig. $3 \mathrm{~b}$. The red dots indicate the observed wavelengths.

respectively. Fe I 6301-6302 А and Ca II $8542 \AA$ were recorded in full Stokes polarimetry. Although the seeing-induced crosstalk is corrected using two liquid crystal modulators and a beam splitter for the temporal and spatial modulation, respectively, a small remaining cross-talk is still present in the Stokes profiles. Since we assumed a Zeeman regime, we have corrected the residual cross-talk by minimising the polarisation signal at the wavelength positions closest to the continuum.

In the magnetic field calculations shown in Sect. 3, we used the frame of the $\mathrm{Fe}_{\mathrm{I}}$ 6301-6302 $\AA$ time series recorded with the best atmospheric conditions. The polarisation signal in Ca II $8542 \AA$ is usually weaker. To increase the signal-to-noise ratio, we computed the median of nine frames selected in a time interval of seven minutes. We chose to apply the median because of its efficacy in reducing the impulse noise (Gonzalez \& Woods 2002).

We estimated the noise levels $(\sigma)$ of Stokes $Q, U$, and $V$ for the wavelength positions closest to the continuum as the standard deviation of the polarisation signals normalised to the continuum intensity, taking into account all the pixels of the SGS. For $\mathrm{Fe}_{\mathrm{I}} 6301-6302 \AA, \sigma$ is $1.5 \times 10^{-3}$ in $Q, U$, and $V$. For Ca II $8542 \AA, \sigma$ is $1.2 \times 10^{-3}$ in $V$ and $1.0 \times 10^{-3}$ in $Q$ and $U$. These polarisation noise values are typical of ground-based filter polarimeters (Lagg et al. 2017). They can be compared with the signal values of Fig. 3. Example profiles of Ca II $8542 \AA$ are displayed in Fig. 1 for three different points within the SGS indicated as 1, 2, and 3 in Fig. 3 b.

At the same time as CRISP, we observed in the blue part of the visible spectrum with CHROMIS, recording along the following spectral lines: 
- Ca II K, at 21 equidistant positions between 3933.1 and $3934.3 \AA$ with $\Delta \lambda=59 \mathrm{~m} \AA$;

- Ca II H, at 21 equidistant positions between 3967.9 and $3969.1 \AA$ with $\Delta \lambda=59 \mathrm{~m} \AA$;

- plus one continuum point at $3999.9 \AA$.

The entire Ca II H\&K profile is scanned in $15 \mathrm{~s}$. At $3933 \AA$, the

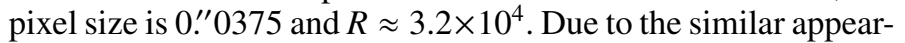
ance of the Ca II H\&K doublet lines, we have decided to show only Ca II K, which forms higher in the chromosphere (Shine et al. 1975).

The data were reduced using the CRISPRED pipeline (de la Cruz Rodríguez et al. 2015) and CHROMISRED pipeline (Löfdahl et al. 2018) for CRISP and CHROMIS data, respectively. Image restoration was performed by employing the Multi-Object Multi-Frame Blind Deconvolution technique (MOMFBD, Löfdahl \& Scharmer 1994; van Noort et al. 2005).

Due to the higher spatial resolution of CHROMIS, we have scaled up the CRISP dataset to the CHROMIS pixel size and spatially aligned it to match the CHROMIS field of view (FOV) by cross-correlating the wide-band images. However, all the calculations involving polarimetry have been performed on nonaligned data, to avoid degradation of the signal-to-noise ratio.

During the same day, part of the SGS was also observed by the Solar Optical Telescope (SOT, Tsuneta et al. 2008) on board Hinode (Kosugi et al. 2007). We employed level 2 data obtained by the SOT spectropolarimeter (SP) running in fast map mode with a pixel size of 0.' 32 . These data were taken between 19:21 and 20:08 UT, so they are not co-temporal with SST observations.

In addition, we used observations with the Helioseismic and Magnetic Imager (HMI, Scherrer et al. 2012) and the Atmospheric Imaging Assembly (AIA, Lemen et al. 2012) on board the Solar Dynamics Observatory (SDO, Pesnell et al. 2012) to study the time evolution of the SGS during its transit from the limb to the disk centre. The full-disk image of the SDO instruments allows us to observe the entire SGS with a spatial resolution of $1^{\prime \prime}$.

\section{Results}

Figure 2 displays the nominal line cores of Fe I $6302 \AA$ (a), Ca II $8542 \AA$ (b), H $\alpha$ (c), and Ca II $\mathrm{K}$ (d) and the blue wings of $\mathrm{H} \alpha$ (e) and $\mathrm{Ca}$ II $\mathrm{K}$ (f). All the images are rotated according to the CHROMIS FOV (panels $d$ and $f$ ). North is indicated by the red arrow and the disk centre by the green arrow. Figure $2 \mathrm{a}$ shows photospheric faculae forming a circular bright network, which appears elliptical because of the projection effects. The black dotted curve helps the reader to locate the bright network. This structure belongs to an active region featuring a small and a large pore. Figures $2 \mathrm{~b}-\mathrm{d}$ show chromospheric fibrils seemingly rooted in the bright annular network, and converging towards the centre of the SGS. The fibrils in the chromospheric images (Figs. 2b-d) show a very similar pattern and single fibrils can be distinguished in all the three panels. The fibril canopy forms a darker area against the active region background and highlights a structure of supergranular size $\left(\sim 50^{\prime \prime}\right)$. Fibrils of the QS look less combed on the southern and eastern sides of the SGS with respect to those on the northern and western sides. They also exhibit a different apparent length. The entire structure recalls a rosette, except a smaller region in the S-E of the SGS, where fibrils are arranged as a chain. Here, one end of the fibrils is rooted in the annular network while the other lies inside the SGS. This second footpoint location is highlighted in panel $\mathrm{b}$ by a white rectangle. This chain is present during the entire observation but it is well visible only in the Ca II $8542 \AA$ dataset.

The different appearance of the fibrils can be further appreciated in the blue wing of $\mathrm{H} \alpha$ (Fig. 2e). On the west side of the SGS, the dark fibrils look thinner and longer and they are rooted in the bright network. On the contrary, the fibrils look shorter and more clustered on the east side. In the blue wing of $\mathrm{Ca}$ II $\mathrm{K}$ (Fig. 2f) we observe bright and thin fibrils, which are known as straws (Rutten 2007) or slender Ca II H fibrils (Jafarzadeh et al. 2017). In our observations, these fibrils are rooted exactly above the bright granules of the active region (Fig. 2a), in agreement with previous publications (Rutten 2007; Pietarila et al. 2009; Jafarzadeh et al. 2017). Moving towards the line centre, the straws progressively lose their sharpness and become partially covered by dark and opaque fibrils. We do not note any remarkable morphological difference between straws of different sides. Straws have been suggested to be the tracer of the magnetic field direction (e.g. Schrijver et al. 1989; Jafarzadeh et al. 2017), thus we would expect a rather vertical field all along the network ring surrounding the SGS.

\subsection{Magnetic topology}

To retrieve the photospheric magnetic field vector of the entire FOV, we performed simultaneous inversion of the Fe I 6301$6302 \AA$ lines using the MINE code (de la Cruz Rodriguez et al., in prep.). MINE is a parallel single component Milne-Eddington (ME) inversion with analytical response functions (Skumanich \& Lites 1987; Orozco Suárez \& Del Toro Iniesta 2007). It can include the $6301 \AA$ and $6302 \AA$ lines in one single inversion following the scheme presented by Orozco Suárez et al. (2010). MINE adjusts the physical parameters of the guessed input model in order to minimise the difference between the observed and synthetic profiles using a standard Levenberg-Marquardt algorithm. The synthetic profiles and the response functions are convolved with the CRISP instrumental profile after each spectral synthesis. Here, we performed the inversion in two runs. In the first run, we assigned the same input model atmosphere to all the pixels. The atmospheric parameters are the total magnetic field strength, the magnetic field inclination, the azimuth, the line-of-sight velocity, the Doppler width, the line opacity, the damping parameter, and source function parameters. Macrotubulence is set to zero. We used a smoothed version of the output model to feed the second run, which produces the final result.

The chromospheric magnetic field is obtained using the weak field approximation from the temporal median of nine Ca II $8542 \AA$ frames. We applied the relations derived in Degl'Innocenti \& Landolfi (2004),

$$
\begin{aligned}
& V(\lambda)=-\frac{\lambda_{0}^{2} e_{0}}{4 \pi m_{\mathrm{e}} c^{2}} \bar{g} B \cos \theta \frac{\partial I}{\partial \lambda} \\
& U(\lambda)=\frac{3}{4} \frac{\lambda_{0}^{4} e_{0}^{2}}{\left(4 \pi m_{\mathrm{e}} c^{2}\right)^{2}} \frac{\bar{G}}{\lambda-\lambda_{0}} B^{2} \sin ^{2} \theta \frac{\partial I}{\partial \lambda} \sin 2 \psi \\
& Q(\lambda)=\frac{3}{4} \frac{\lambda_{0}^{4} e_{0}^{2}}{\left(4 \pi m_{\mathrm{e}} c^{2}\right)^{2}} \frac{\bar{G}}{\lambda-\lambda_{0}} B^{2} \sin ^{2} \theta \frac{\partial I}{\partial \lambda} \cos 2 \psi
\end{aligned}
$$

where $\lambda_{0}$ is the nominal spectral line centre, $e_{0}$ the electron charge, $m_{\mathrm{e}}$ the electron mass, $c$ the speed of light, $\bar{g}$ the effective Landé factor, $B$ the magnetic field strength, $I$ the intensity, $\psi$ the azimuthal angle of the magnetic field, and $\theta$ the 

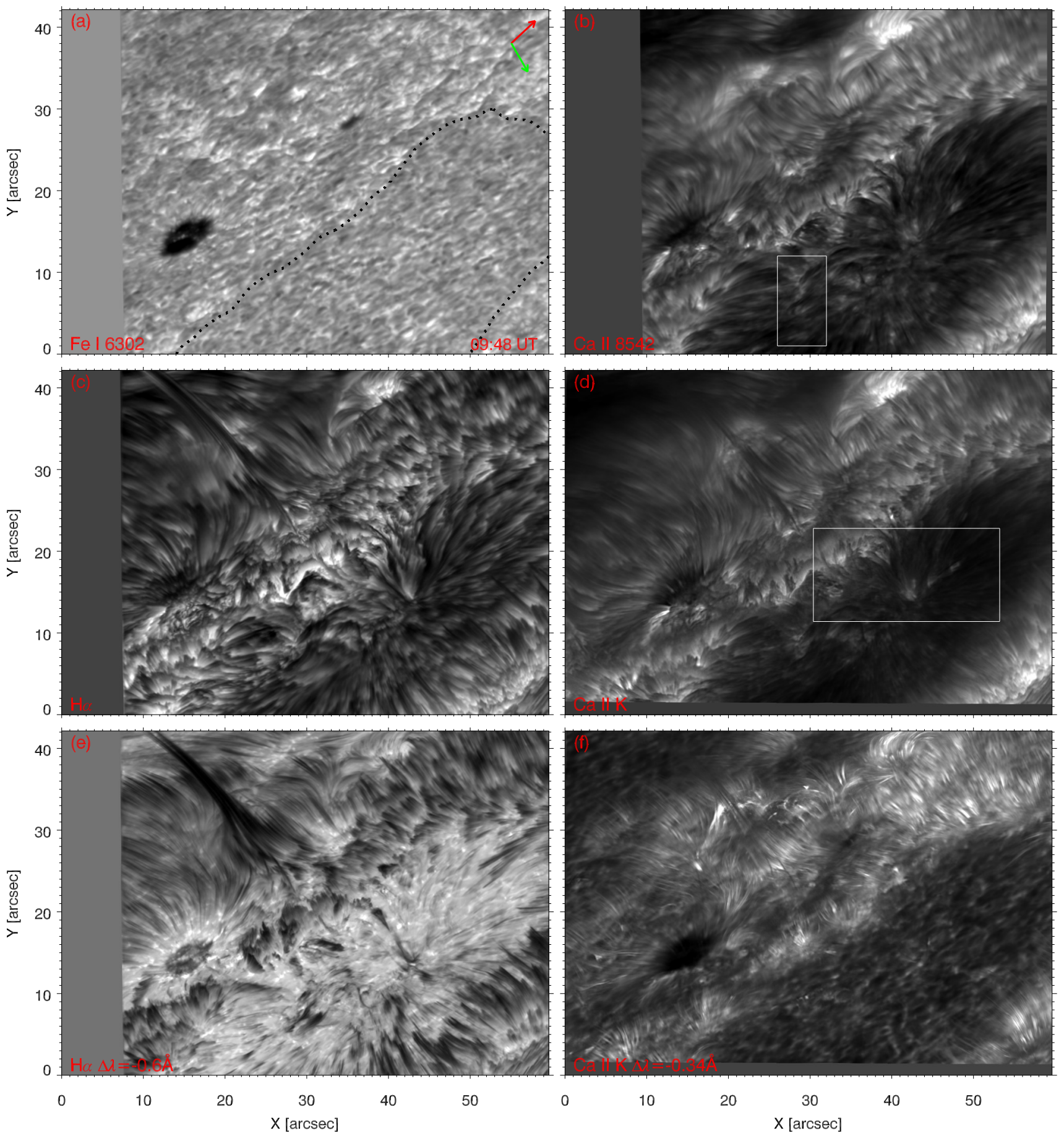

Fig. 2. Nominal line core intensity map of Fe I $6302 \AA$ (panel a), Ca II $8542 \AA$ (panel b), H $\alpha$ (panel c), and Ca II K (panel d) and blue wings intensity of $\mathrm{H} \alpha$ (panel e) and Ca II $\mathrm{K}$ (panel $f$ ) at $\Delta \lambda=-0.6 \AA$ and $\Delta \lambda=-0.34 \AA$, respectively. The images are spatially and temporally co-aligned according to the CHROMIS field of view. The black dotted curve identifies the bright network. The white rectangle in panel $b$ corresponds to the one shown in Fig. 3b while the rectangle in panel $d$ highlights the region shown by Fig. 9. The red arrow indicates the north and the green arrow the disk centre. The temporal evolution is shown in a movie in the online edition.

inclination. The function $\bar{G}$ depends on the angular momenta of the transition levels and its full expression can be found in Degl'Innocenti \& Landolfi (2004). Equation (1) holds true along the entire spectral line profile, while Eqs. (2) and (3) better apply in the wings. Therefore, we omitted the nominal line core in the calculations of the transversal component of the magnetic field. The two components of the magnetic field (longitudinal and transversal) and the azimuth are obtained by least-squares minimisation.
The transversal component of the magnetic field is affected by the Zeeman ambiguity regardless of the technique employed to calculate the field. The ambiguity has to be removed in order to rotate the reference system from line of sight to local solar coordinates (LSC). In regions of relatively strong photospheric field, such as our active region and annular network, the ambiguity can be solved by using standard methods. We applied the minimum energy method (MEM, Metcalf 1994), using the implementation of Leka et al. (2014). Solving the ambiguity in 

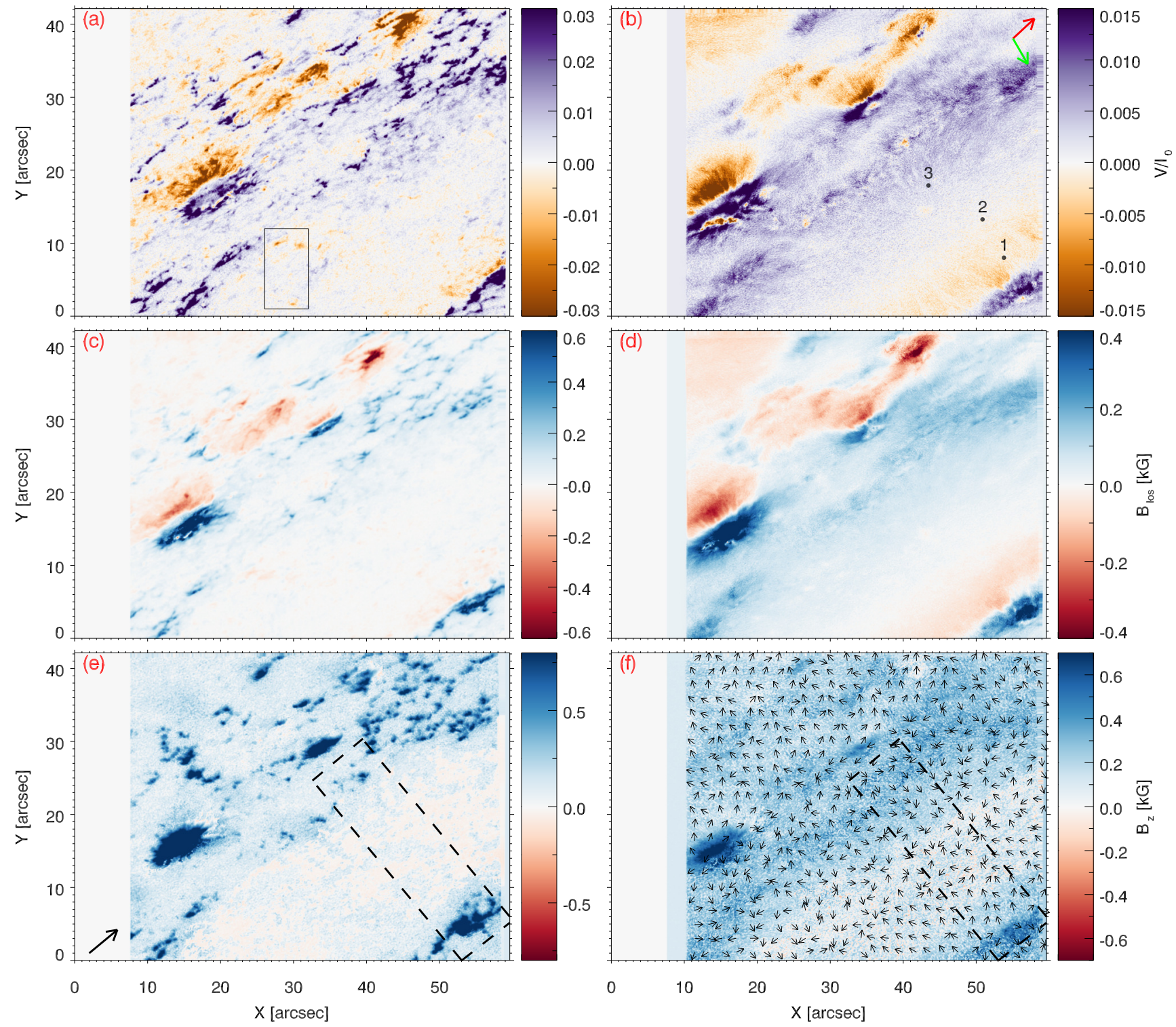

Fig. 3. Polarisation and magnetic field maps: circular polarisation of Fe I $6302 \AA$ at $\Delta \lambda=-0.08 \AA$ (panel a) and Ca II $8542 \AA$ at $\Delta \lambda=-0.29 \AA$ (panel b). Line-of-sight magnetic field strength obtained from the Fe I 6301-6302 ̊ (panel c) and Ca II $8542 \AA$ lines (panel d). Vertical magnetic field strength for the $\mathrm{Fe}_{\mathrm{I}}$ 6301-6302 $\AA$ (panel e) and $\mathrm{Ca}$ II $8542 \AA$ lines (panel f). In panel $b$, the red arrow indicates the north and the green arrow the disk centre. The points indicated with 1,2, and 3 refer to Fig. 1. The dashed rectangle and the black arrow of panel e refer to Fig. 4 . The black arrows in panel $f$ indicate the direction of the horizontal field.

the portion of the QS contained in the SGS is more challenging because the polarisation signal is lower than in the active region and there is no clear indication for a preferred field direction. Previous studies have shown that the field in the QS tends to be horizontal (e.g. Lites et al. 2008; Bommier et al. 2009; Bommier 2011; Orozco Suárez \& Bellot Rubio 2012), in particular Bommier (2011) noticed that the stronger the magnetic field, the larger is the angle between the solar surface and the field lines. Considering this remark, we have applied the following strategy: first, we calculated the magnetic field vector in the LSC for the two possible azimuthal directions and, using these values, the two possible inclinations. Then, we imposed these conditions to select the most likely value:

1. Observing that for our large heliocentric angle the horizontal magnetic field in the LOS component $\left(B_{\mathrm{x}}^{\text {los }}\right)$ is the closest in magnitude to the LSC longitudinal field $\left(B_{\mathrm{z}}\right)$, then, when $B_{\mathrm{x}}^{\text {los }}>500 \mathrm{G}$, we choose the azimuth that maximises the inclination.
2. Close to the limb, the largest part of the circular polarisation signal is produced by horizontal magnetic field. Thus, the lineof-sight magnetic field (Fig. 3c) provides an indication of the actual horizontal magnetic field direction in the LSC, without ambiguity. Since the magnetic field in the pore is positive and radial, we can infer that, if $\mathrm{B}$ is negative along the LOS, the horizontal field is directed from the disk centre towards the SGS centre. Otherwise, the field is directed from the limb towards the disk centre. Thus, for those pixels where $B_{\mathrm{x}}^{\text {los }}<500 \mathrm{G}$, we impose that, if $B_{\mathrm{z}}^{\text {los }}$ is positive, the azimuth, which in our case ranges between $0^{\circ}$ and $180^{\circ}$, is smaller than $90^{\circ}$.

The results have been compared to the $B_{\mathrm{z}}^{\text {los }}$ map of the same SGS observed by HMI five days later, which was close to disk centre. The general polarity distributions are consistent with each other for both the active region and the QS.

Figure 3a shows the Stokes $V$ signal of Fe I $6302 \AA$. The signal is stronger along the boundaries of the SGS while there 

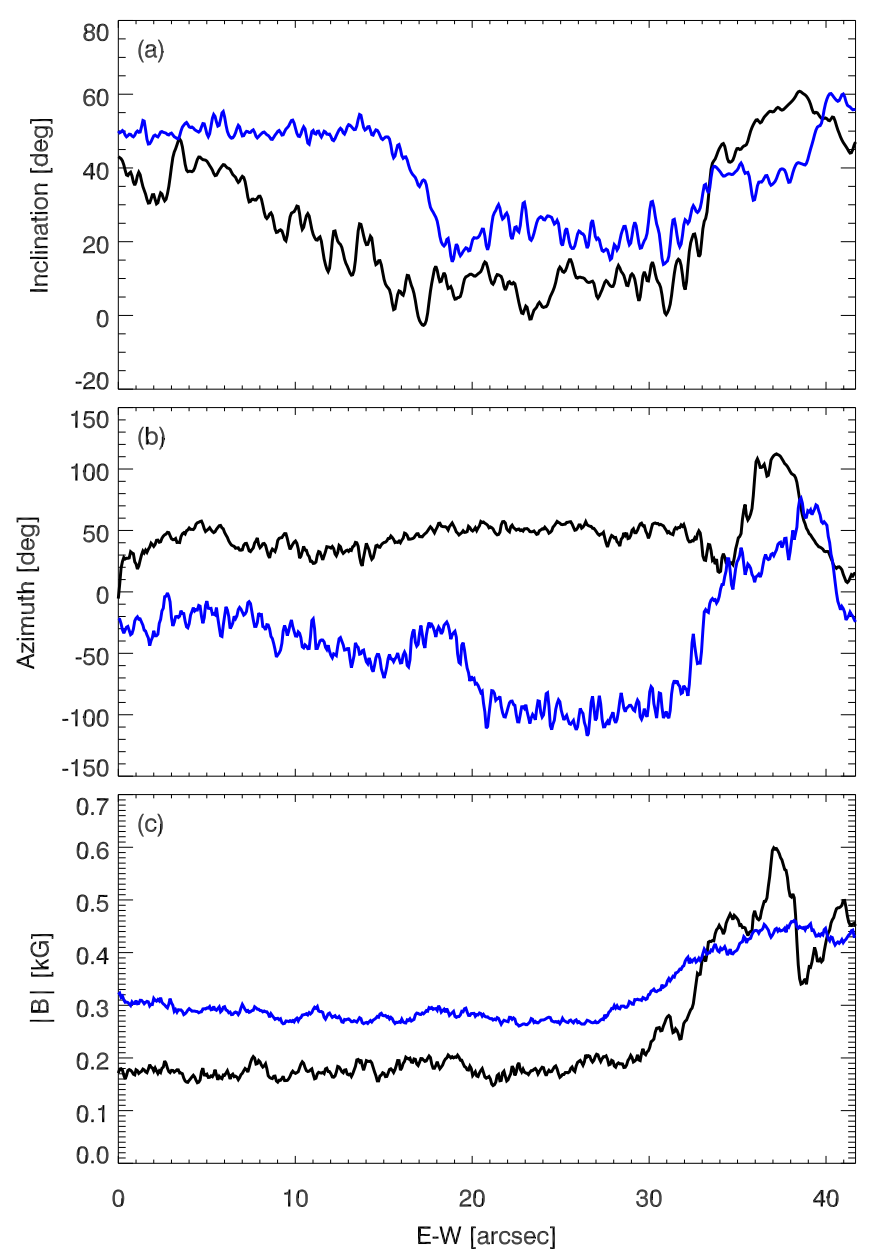

Fig. 4. Value of the inclination (panel a), azimuth (panel $b$ ), and strength (panel c) of the magnetic field obtained from Fe I 6301-6302 A (black curve) and Ca II $8542 \AA$ data (blue curve) averaged inside the dashed box of Fig. 2e along the direction indicated by the black arrow of Fig. 2e. Vertical magnetic field corresponds to an inclination of $90^{\circ}$. The zero azimuth is defined with respect to the westerly direction (counterclockwise).

is a weaker pattern of internetwork within the SGS. The black inset indicates the second footpoint of the fibrils arranged as a chain. The Stokes $V$ signal in this region is slightly higher than in the rest of the inner SGS, probably affecting the fibril arrangement. Figure $3 \mathrm{c}$ shows the LOS magnetic field retrieved by $\mathrm{ME}$ inversion. The vertical component of magnetic field in the LSC is displayed in Fig. 3e. From this map, we can read that the active region and the network ring around the SGS are positive and the SGS has a weak mixed polarity. We selected the pixels in the dashed box of Fig. 3e and calculated the mean inclination by averaging the pixels along the direction shown by the black arrow. The black curve of Fig. 4 a represents the average inclination of the SGS structure, with $0^{\circ}$ equal to a horizontal magnetic field. The network ring is on average more vertical than the QS inside the SGS. In the same way, we plotted the averaged azimuth in Fig. 4b (black curve). The zero coincides with the longer axis of the dashed box directed westwards. Fig. 4c shows the average value of the magnetic field strength calculated as in panel a and b. The photospheric field (black) is constant within the SGS and rapidly increases at the supergranular boundaries.

The same quantities of the left column of Fig. 3 are shown for Ca II $8542 \AA$ on the right. Figure 4d displays the longitudinal magnetic field component obtained in weak field approximation. To choose the direction of transversal field, we applied the second argument employed for the Fe I $6302 \AA$ azimuth solution. The correct azimuth is used to rotate the magnetic field vector in the local solar reference frame. Figure $3 \mathrm{f}$ shows the vertical magnetic field in the LSC $\left(B_{\mathrm{z}}\right)$.

We calculated the chromospheric field inclination in the same way as the photosphere and displayed it as a blue curve in Fig. 4a. The right side of the curve (west side of the network) corresponds to an extended concentration of strong magnetic field. Here, the photospheric magnetic field is more vertical. The blue curve in Fig. 4a shows that the magnetic field obtained by the weak field approximation tends to be rather horizontal on the centre of the SGS, while it is more inclined on the boundaries. In Fig. 4b, we show the average azimuth (blue curve). On the west side of the SGS, this is $-110^{\circ}$ while on the east side it is about $-20^{\circ}$. The azimuth is also indicated by the black arrows in Fig. 3f. These arrows show that on the west side of the box, a part of the field lines converges from the north towards the centre of the SGS with an azimuth close to $-90^{\circ}$. Another set of field lines converges from the west with an azimuth of roughly $-135^{\circ}$. This is in agreement with the direction of the corresponding fibrils (compare to Fig. 2). Thus, the average azimuth $\left(\sim-110^{\circ}\right)$ in the west side of the box (Fig. 4b) represents the average between these two main directions. The azimuth values on the east side of the box $\left(\sim-20^{\circ}\right)$ and on the west side $\left(\sim-110^{\circ}\right)$ indicate that the horizontal field tends to point towards the inner part of the SGS. The blue curve of Fig. $4 \mathrm{c}$ represents the average chromospheric magnetic field strength. Its value above the SGS is rather constant and, similarly to the photosphere, it increases above the boundaries.

A co-temporal observation with SDO (Fig. 5) shows that no structure is visible above the SGS in $171 \AA$, except for a weakly bright structure following the fibrils arrangement, which might be a hint of a low-lying canopy. The network is bright and several vertical plumes fan out from the outer part of the ring, where the field is more concentrated.

\subsection{Flux imbalance}

The vertical magnetic field allows us to compute the total photospheric flux inside the SGS and along its annular network. The general magnetic flux expression,

$\Phi(B)=\int_{S} B \cdot \mathrm{d} s$,

can be discretised into an expression for the positive flux, $+\Phi(B)$, and another for the negative flux, $-\Phi(B)$,

$\pm \Phi(B)=\sum_{x} \sum_{y} \pm B_{z}(x, y) \Delta A$,

where $\Delta A$ is the pixel area. The positive (negative) flux is calculated by summing over all the pixels where $B_{\mathrm{z}}$ is positive (negative).

The vertical magnetic field in Fig. 3a has been aligned to the FOV of CHROMIS to match the intensity maps of Fig. 2, but to calculate the flux we used the non-aligned longitudinal field map, shown in Fig. 6a. However, the FOV of CRISP does not capture the entire SGS and the northern and southern sides of the cell are missed.

Therefore, we have decided to employ magnetograms of the same SGS observed by other instruments. Hinode/SP data display a large fraction of the SGS ten hours after our observing 

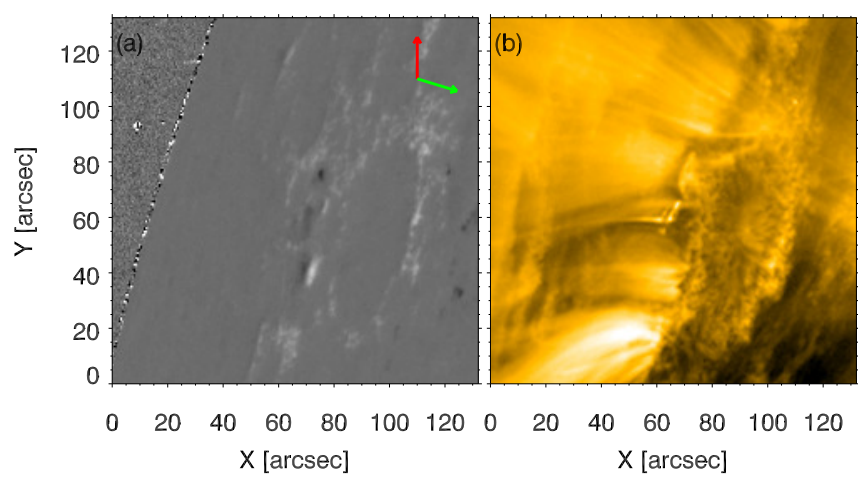

Fig. 5. Left panel: SDO/HMI line of sight magnetogram with a larger field of view respect to Fig. 3 and different orientation. The red arrow indicates the north and the green arrow the disk centre. Right panel: SDO/AIA $171 \AA$ image with the same FOV as panel $a$. Both images are co-temporal to images in Fig. 2.

run at the SST and show the missing structure of the CRISP data. To avoid projection effects, we also used the HMI magnetogram, which was obtained close to disk centre on April 25. After five days, we still observe a similar unipolar region, but it is impossible to directly compare with SST data. For all the data, we manually created a mask to choose the inner SGS and another one to select the annular network at the boundaries. Both masks are indicated by orange contours in Figs. 6a, b, and d. As can be seen in panel a, the two pores within the dotted lines were intentionally excluded from the outer mask. Table 1 summarises the results of the flux calculations. The in superscript stands for the QS region within the SGS while out is for the area enclosed by the two orange contours.

Although we cannot compare the results inferred for each dataset because of the different FOV and resolution, we have nonetheless three independent measurements of flux disequilibrium. The annular network has a strong positive flux that is not balanced by the negative flux of the entire SGS. In contrast, the positive and negative fluxes are closer to equilibrium in the inner region, although we observe an excess of positive polarity.

The different order of magnitude obtained from the inner flux calculations is likely due to the different spatial resolution of each instrument. The Hinode/SP network flux has the same order of magnitude as the lower spatial resolution SDO/HMI, but we have to consider that the Hinode/SP FOV lacks a non-negligible fraction of the eastern side of the SGS. Therefore we expect the actual total value to be higher, as well as for the SST/CRISP network flux.

Considering line core intensity filtergrams in Ca II $8542 \AA$, we can choose a smaller mask enclosing the region where fibrils are bending towards the SGS centre. This choice (not shown), however, decreases the value of the positive flux by $20 \%$ only, which is insufficient to balance the positive annular ring flux.

All the three cases (Figs. 6a, b, and d) show that the negative and positive field of the entire SGS are significantly unbalanced and the small negative polarity patches in the inner region are not enough to balance the positive network field. The SDO/HMI coronal image in $171 \AA$ shown in Fig. 5 suggests that part of the positive flux might follow the locally open field lines rooted in the network and connect with negative polarity outside the FOV.

There is an asymmetry in the magnetic field concentration between the east and west side of the SGS. This asymmetry is exemplified in Fig. 6, where we plot the longitudinal magnetic field averaged along the N-S direction (red arrow in Fig. 6a) as a function of E-W direction (black arrow). The increasing

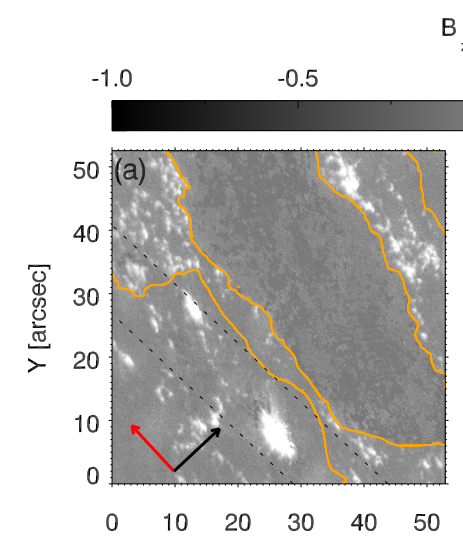

$B_{z}[k G]$

0.0
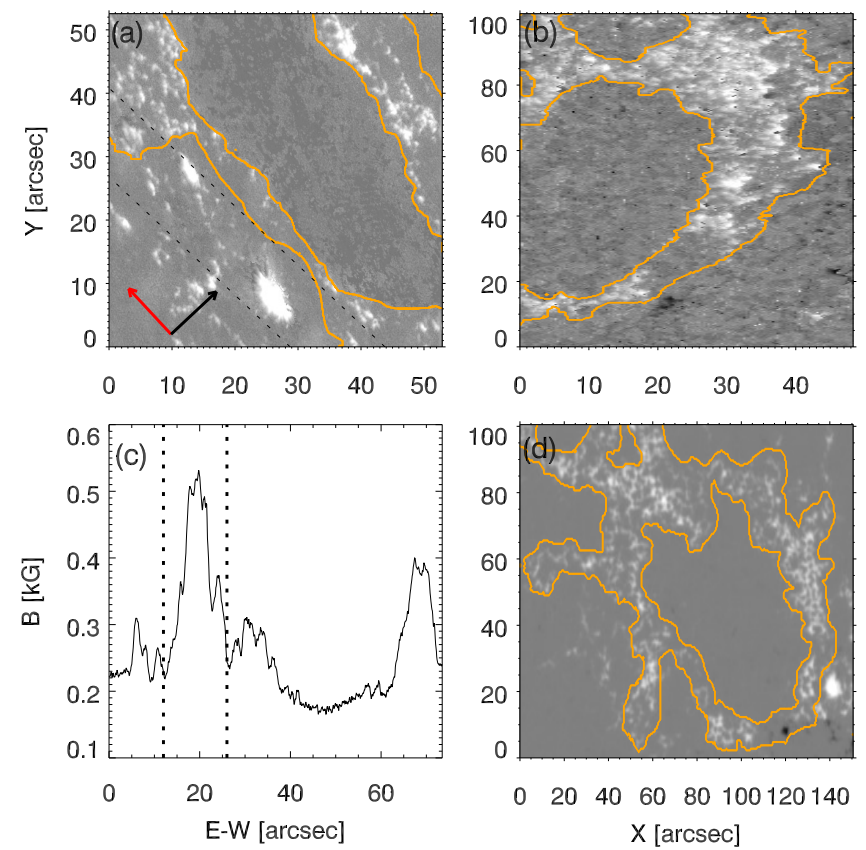

Fig. 6. Vertical magnetic field map obtained with CRISP (panel a), Hinode/SP (panel $b$ ), and SDO/HMI (panel $d$ ). The red arrow indicates the north and the black arrow the E-W direction. In panels $b$ and $d$, the north coincides with the top side of the image. The orange contour indicates the masks employed for the flux calculation. Panel $c$ : average magnetic field strength along the E-W direction calculated from the CRISP data. The dotted lines in panels $a$ and $c$ highlight the region containing the pores.

values on the $x$-axis go from $\mathrm{E}$ to $\mathrm{W}$. The region between the two dotted lines indicates the location of the pores. The network field enhancement is visible around $X=20^{\prime \prime}$ on the east side and around $X=67^{\prime \prime}$ on the west side. The east side is on average weaker and less concentrated. The same asymmetry was reported by Langfellner et al. (2015) in a statistical study of more than 3000 supergranules observed close to disk centre. The different appearance of the SGS fibrils shows the same E-W asymmetry. According to the scenario of the magneto-acoustic origin of type-I spicules (e.g. De Pontieu et al. 2004, 2007), the length of the SGS fibrils should be dependent on the magnetic field inclination (Zhugzhda \& Dzhalilov 1984; Jefferies et al. 2006). Consequently, we would expect a more horizontal field on the west side, where the fibrils look longer. The blue curve of Fig. 4a does not contradict this scenario. However, close to limb it is impossible to discern whether there is an actual physical correlation between the two asymmetries or whether it is a projection effect.

\subsection{LOS velocity}

The online animation of Fig. 2 shows a dynamic atmosphere above the SGS. Thanks to multi-wavelength observations, we can provide velocity maps at different atmospheric heights.

Figure 7 displays the LOS velocity map of the photosphere (panel a) and chromosphere (panel b) at 09:48 UT. The photospheric map was obtained from ME inversion and it was calibrated using as reference the velocity inside the pore, in a similar way to that proposed by Beckers (1977). 
Table 1. Positive and negative flux calculated inside the SGS $\left(\Phi_{B}^{\mathrm{in}}\right)$ and along the annular network $\left(\Phi_{B}^{\text {out }}\right)$ for each of the instruments shown in Figs. 6a, b, d.

\begin{tabular}{|c|c|c|c|c|c|c|c|c|}
\hline \multirow[t]{2}{*}{ Instrument } & \multicolumn{2}{|c|}{$\Phi_{B}^{\mathrm{in}}(\mathrm{Mx})$} & \multicolumn{2}{|c|}{$\Phi_{B}^{\text {out }}(\mathrm{Mx})$} & \multicolumn{2}{|c|}{$A^{\text {in }}\left(\mathrm{cm}^{2}\right)$} & \multicolumn{2}{|c|}{$A^{\text {out }}\left(\mathrm{cm}^{2}\right)$} \\
\hline & + & - & + & - & + & - & + & - \\
\hline SDO/HMI & $4.6 \times 10^{23}$ & $1.1 \times 10^{23}$ & $5.4 \times 10^{25}$ & $1.1 \times 10^{22}$ & $8.9 \times 10^{18}$ & $5.2 \times 10^{18}$ & $2.3 \times 10^{19}$ & $5.2 \times 10^{17}$ \\
\hline Hinode/SP & $2.7 \times 10^{24}$ & $1.8 \times 10^{24}$ & $4.2 \times 10^{25}$ & $1.5 \times 10^{23}$ & $3.9 \times 10^{18}$ & $3.4 \times 10^{18}$ & $7.7 \times 10^{18}$ & $8.8 \times 10^{17}$ \\
\hline SST/CRISP (Fe I) & $5.8 \times 10^{25}$ & $1.3 \times 10^{25}$ & $2.5 \times 10^{26}$ & $7.57 \times 10^{21}$ & $3.2 \times 10^{18}$ & $2.5 \times 10^{18}$ & $4.3 \times 10^{18}$ & $7.9 \times 10^{16}$ \\
\hline
\end{tabular}

Notes. $A^{\text {in }}$ represents the total amount of positive (+) and negative (-) pixels in the inner mask, multiplied by the single pixel area. The same for $A^{\text {out }}$ in the outer mask.
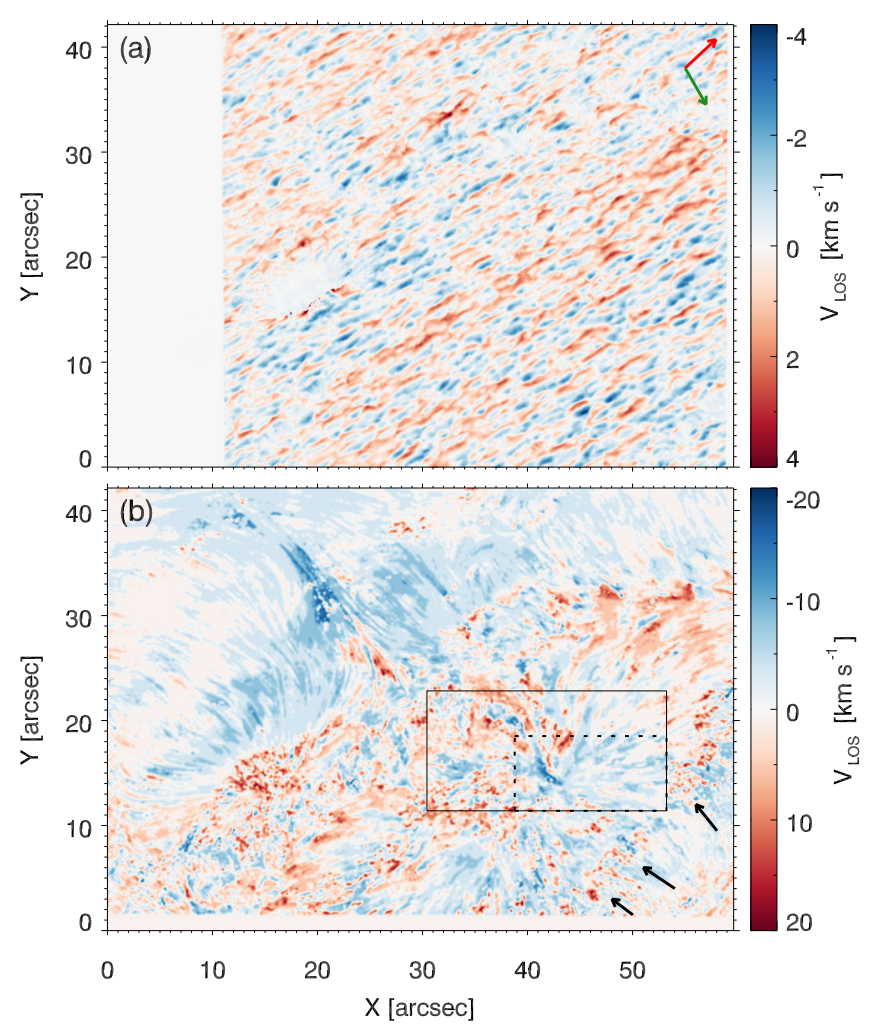

Fig. 7. Top panel: photospheric LOS velocity obtained with MilneEddington inversion of $\mathrm{Fe}_{\mathrm{I}}$ 6301-6302 ̊.. Bottom panel: chromospheric LOS velocity obtained from the $K_{3}$ features Doppler shift. The black solid rectangle highlights the region shown in Fig. 9, the dotted rectangle the region in Figs. 8 and 10.

The LOS velocity map mostly shows the LOS projection of the granular flows. We do not observe any anomalies either for the time step shown in Fig. 7a, or for the entire time series.

Figure $7 \mathrm{~b}$ displays the LOS velocity obtained from the Doppler shift of the Ca II $\mathrm{K}$ core $\left(K_{3}\right)$, which is a good diagnostic tool for the upper chromospheric velocity (Bjørgen et al. 2018).

We preferred to compute the LOS velocities from the $K_{3}$ peak because $\mathrm{Ca}$ II $\mathrm{K}$ is less affected by thermal broadening than $\mathrm{H} \alpha$ and it does not suffer from the inverse C-shape that affects Ca II $8542 \AA$.

To identify the $K_{3}$ feature, we developed an algorithm that automatically searches for the line core of Ca II K. It uses the definition of $K_{3}$ based on a typical QS profile of Ca II $\mathrm{K}$ where the $K_{3}$ feature corresponds to the intensity minimum confined between two emission peaks, known as $K_{2 \mathrm{v}}$ on the blue wing and $K_{2 \mathrm{r}}$ on the red wing (Hale \& Ellerman 1904). However, this definition is not suitable for some spectral profiles, such as those emerging from strong magnetic field regions like the pores and the annular network, which exhibit a single emission peak. There, the algorithm identifies $K_{3}$ as the wavelength corresponding to the minimum intensity.

We calculated the chromospheric LOS velocity using the Doppler shift of $K_{3}$, wavelength-calibrated with the atlas obtained from the Fourier Transform Spectrometer at the National Solar Observatory on Kitt Peak (Neckel 1999). To perform a precise velocity calibration, we also considered the centre-to-limb variation of the LOS velocity. From the nominal line centre value of the atlas, we estimated a velocity offset of about $-600 \mathrm{~m} \mathrm{~s}^{-1}$, which is comparable to the results of de la Cruz Rodríguez et al. (2011). All the velocities are also corrected for the blue-shift produced by the solar rotation $\left(\sim 1.8 \mathrm{~km} \mathrm{~s}^{-1}\right)$.

Interpretation of the entire map is difficult due to the large observing angle and the different inclination of each fibril. It is not possible to detect a clear bulk motion along them, but as a general trend we notice that the structure closer to disk centre is more blue-shifted while, on the other side, profiles tend to be more red-shifted. The black arrows in Fig. 7b mark the transition between the apparently vertical and bright fibrils and the darker and horizontal ones, as we observed in Ca II $8542 \AA$ and Ca II K line cores.

In the convergence point of most of the fibrils (around $X=$ $42^{\prime \prime}, Y=14^{\prime \prime}$ ), a significantly blue-shifted structure appears. Comparing with Figs. 2b, c, and d, we recognise part of a fibril.

This strong blue-shift is visible during the entire observation ( $20 \mathrm{~min}$ ) at the same location (see Fig. 8) and its shape changes during the observation.

It is non-trivial to establish whether this blue-shift is an actual upflow or a downflow observed along a structure that is inclined towards the limb.

Figure 9 shows a close-up of the centre of the SGS, which is enclosed by a white rectangle in Fig. 2 d. Figure 9a shows the intensity in the nominal line centre of $\mathrm{Ca}$ II $\mathrm{K}$ at the same time step used to calculate the Dopplergram shown in Fig. 7. A bright structure appears at the centre. Figure $9 \mathrm{~b}$ displays the intensity map corresponding to the wavelength position that the algorithm identifies as $K_{2 \mathrm{r}}$ peak, at the same time step as panel a. Most of the pixels have a well-defined Ca II K intensity profile and the $K_{2 \mathrm{r}}$ peak is successfully identified. However, there are some pixels where they have eluded detection (masked in light green). The $K_{2 r}$ intensity shows the same enhancement of Fig. 9a. To exclude the possibility that the chosen frame has a special appearance, we display the same region at a different time step (panel c). Here we observe an apparent vertical fibril arrangement with a brighter bottom. The online animation of Fig. 2 shows that this intensity enhancement in the core of the SGS is always present. 




Fig. 8. Enlargement of the dotted rectangle of Fig. $7 \mathrm{~b}$ at three different time steps: 09:43 UT (left panel), 09:52 UT (middle panel), and 09:59UT (right panel).
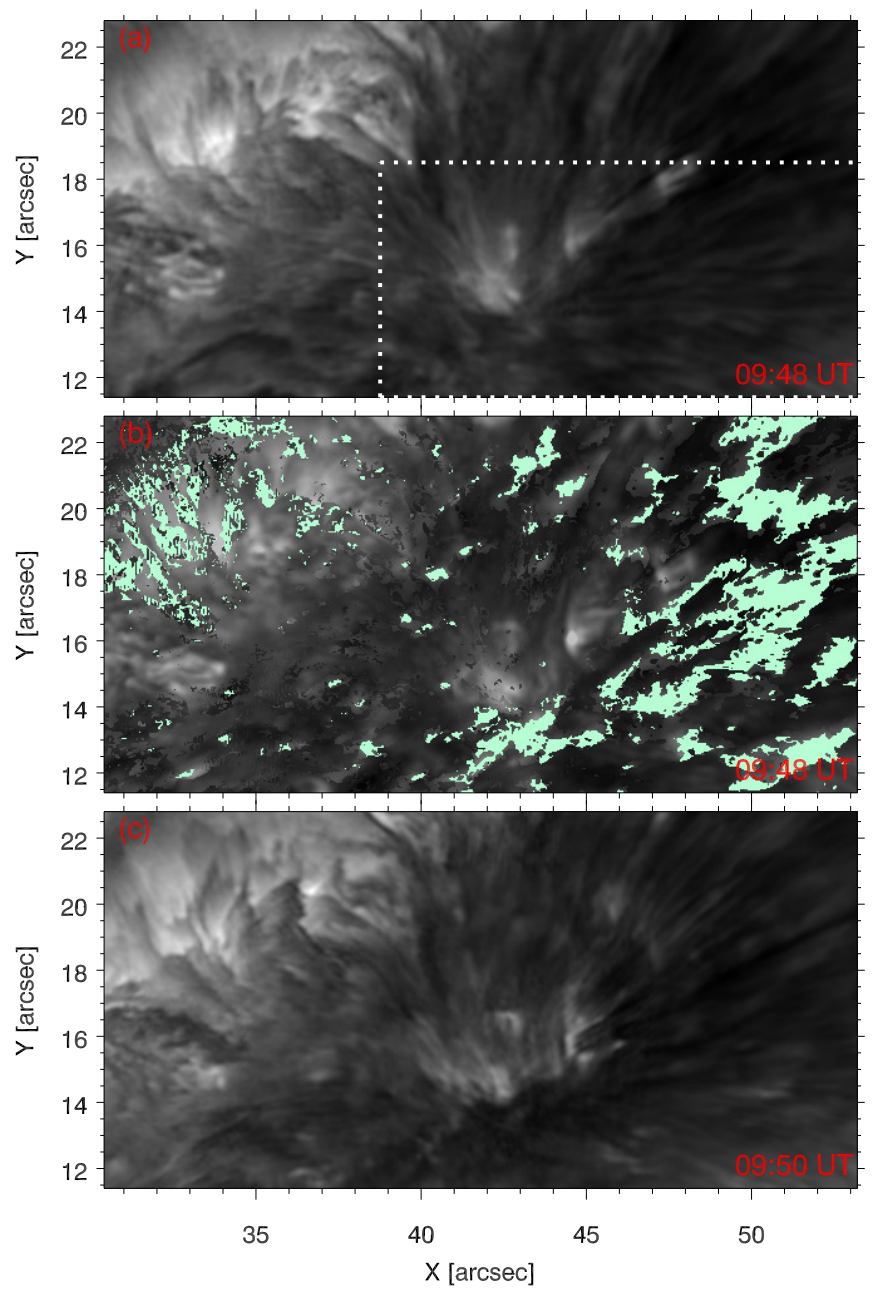

Fig. 9. Close-up of the white rectangle shown in Fig. 2 of the Ca II $\mathrm{K}$ nominal line centre (panel $a$ ) and the $K_{2 \mathrm{r}}$ intensity (panel $b$ ). The light green mask represents the pixels where the $K_{2 \mathrm{r}}$ feature cannot be detected. Panel $c$ : same field of view in the nominal line centre of $\mathrm{Ca}$ II $\mathrm{K}$ at a different time (09:50 UT). All the images are corrected with a gamma factor of 0.5 .

To understand the origin of the bright SGS centre, we analysed the profiles emerging from the region enclosed by the dotted rectangle in Fig. 9a We applied the $k$-mean algorithm to cluster the spectral profiles in four different typical profiles.

The results of the clustering are displayed in Fig. 10. The image is shown with reversed intensity contrast to help the reader to identify the colour contours. Each colour contour contains the pixels where the profiles are more similar to the average profile of the same colour. The bright centre is described by only one typical profile (azure). This typical profile does not exhibit
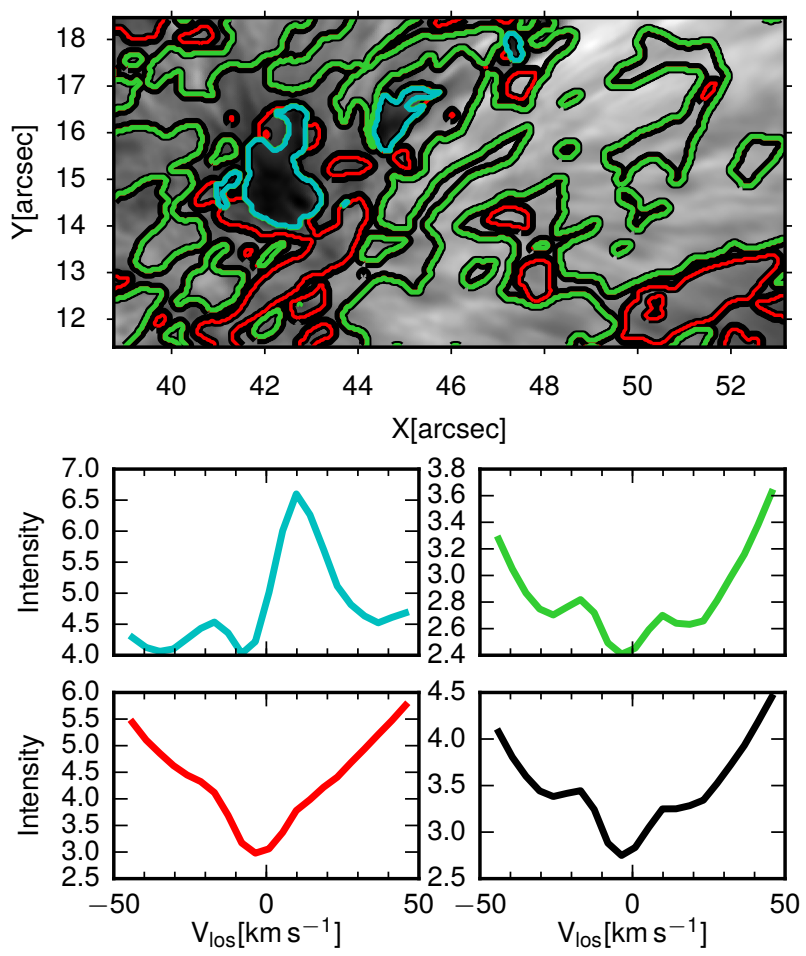

Fig. 10. $K$-mean clustering of the spectral profiles of the inset of Fig. 9a. Each contour delimits the area where the profiles are closer to the corresponding coloured average profile. The upper image has reversed intensity and the intensity scales of the last four panels are in units of $10^{-7} \mathrm{erg} \mathrm{cm}^{-2} \mathrm{~s}^{-1} \mathrm{~Hz}^{-1} \mathrm{ster}^{-1}$. The Y-axes have different scales.

a strong blue-shift as retrieved in the velocity map because it is an average on many different profiles. The pixels belonging to the centre of the SGS exhibit a significant asymmetry of the $K_{2 \mathrm{r}}$ feature. The strongly enhanced $K_{2 \text { r }}$ peak and the blue-shift are the cause of the bright fibrils observed in the nominal line centre of Ca II K. The clustering results are in agreement with $K_{2 \mathrm{r}}$ intensity map. The rest of the pixels are labelled with typical QS profiles (green and black contour) or complete absorption profiles (red). Complete absorption profiles are only observed in the QS portion of the FOV.

\subsection{Evolution}

SST observations do not last long enough to catch the longterm evolution of the SGS. To detect any remarkable activity connected with the SGS in the following days, we used coobservations with SDO. The left column of Fig. 11 shows the time evolution of the SGS magnetogram. We can see that the day after the observation (panel c), partly due to projection effects, a more positive network is visible inside the SGS and two days 


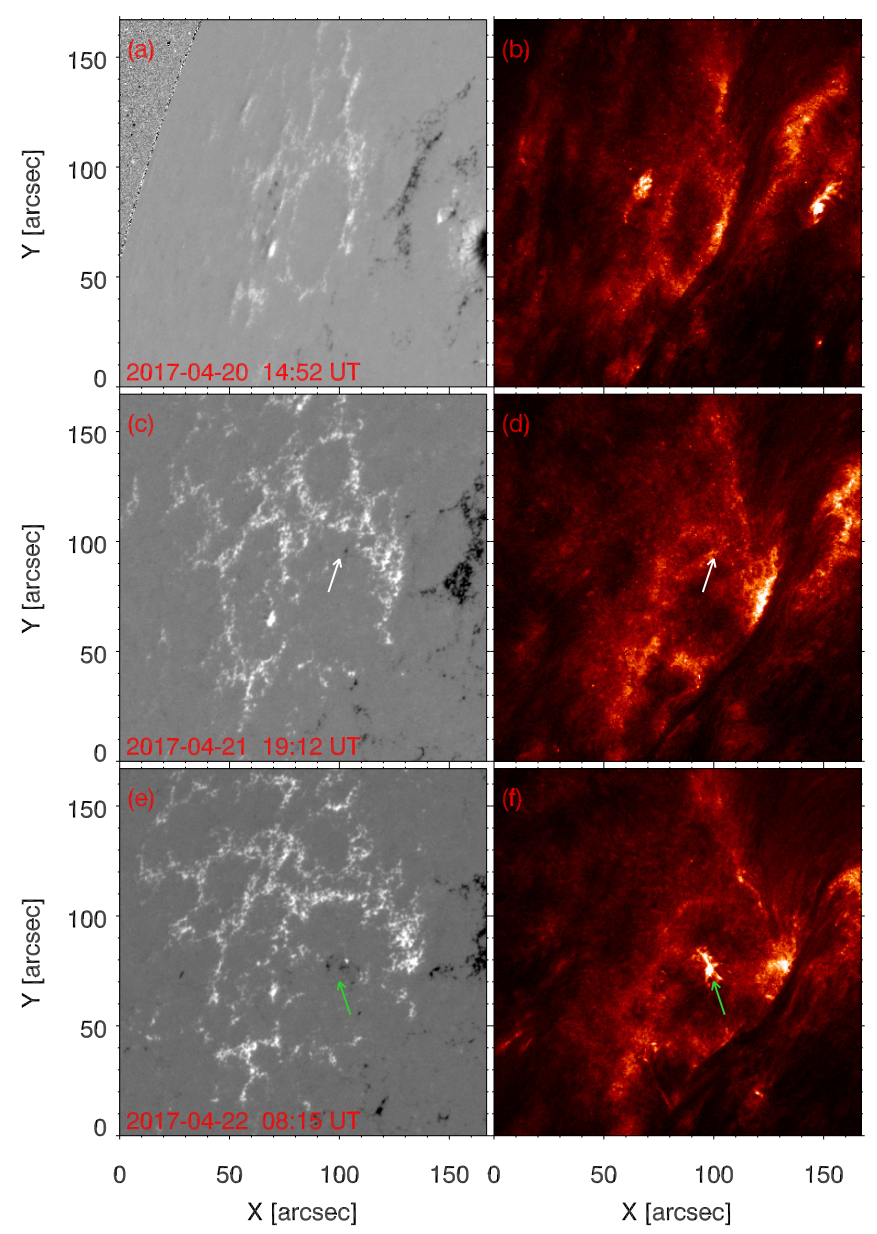

Fig. 11. Left column: HMI line-of-sight magnetogram of the super granular structure. Right column: AIA 304 images for the same field of view as the left column. First row: data acquired on the same day of CRISP observations (panels $a$ and $b$ ). Middle and last row: data obtained one (panels $c$ and $d$ ) and two (panels $e$ and $f$ ) days later. The green and white arrows indicate flux emergence events. The north direction coincides with the top side of the panels.

after (panel e), we can eventually distinguish some magnetic cells converging in its centre.

A small flux emergence appears in the north (see the white arrows), but, according to the co-temporal image of AIA 304 channel (panel d), no significant chromospheric effect is associated with it. Two days after (panel e), we observe a more extended flux emergence in the middle of the SGS (green arrow). The corresponding AIA 304 image (panel f) shows a coherently bright structure lasting for almost 30 minutes.

Overall, the time evolution (Sect. 3.4) and the velocity distribution (Sect. 3.3) indicate the centre of the SGS as a favourable location for dynamic events.

\section{Discussion and conclusions}

We retrieved the local magnetic field in the photosphere and chromosphere, applying respectively $\mathrm{ME}$ inversion techniques and the weak field approximation. Using the Fe I 6301-6302 $\AA$ longitudinal magnetogram, we estimated the flux at the photosphere. The field within the SGS is close to polarity balance with an excess of positive polarity, while the network flux is significantly unbalanced. Even though the annular network belongs to a rather active area, the results are in agreement with the flux disequilibrium found in the QS (e.g. Gošić et al. 2014). The largest fraction of magnetic field in the annular network is positive and it is not balanced by negative field at the boundaries nor inside the SGS. Part of the flux might follow the vertical coronal plumes rooted in the annular network and possibly connect with negative polarity outside the FOV.

Figures $3 \mathrm{f}$ and $4 \mathrm{a}$ show a rather horizontal chromospheric magnetic field above the SGS. This field is on average directed towards the centre of the structure (Figs. 3f and 4b). This configuration is in agreement with the idea of a low-lying canopy field, also suggested by chromospheric images (Fig. 2) and the 171 AIA image (Fig. 5b). Limited by the vertical magnetic field map (Fig. 3f), we cannot clearly see whether the canopy bends upwards. However, if all the magnetic field lines in the centre of the SGS were negative and bent downwards, there would be a significant concentration of negative field in the photosphere. Since we do not detect it, and the average field is positively inclined, we suggest that a large part of the chromospheric field lines point upwards, while the remaining return to the surface, as displayed in Fig. 12. These models represents a section of the SGS along the direction disk-centre to limb. The boundaries are indicated by the plus sign. Above the annular network, the field lines are rather vertical, while they bend and form a sort of canopy above the SGS. The models of Fig. 12 show the large-scale behaviour of the magnetic field based on the popular, but not entirely accepted, magnetic canopy model. The magnetic field vector that we retrieved from CaII $8542 \AA$ confirms the inclination measured in the quiet chromosphere by Lagg et al. (2009) and is in agreement with the supergranular topology in the model of WedemeyerBöhm et al. (2009). However, the actual sub-canopy magnetic field configuration is probably more intricate than the models in Fig. 12. The low-lying fibril canopy, for instance, is indicative of a more complex topology, where the QS is strongly connected to the annular network, as proposed by Schrijver \& Title (2003) and suggested also for the chain configuration by Reardon et al. (2011).

The fibril arrangement in the SGS is reminiscent of a large rosette structure. Despite being morphologically similar, they form at different locations. The point at which fibrils converge in rosettes is usually observed at the vertices of supergranules while the SGS core lies in the centre of the supergranular cell. As a consequence, the rosette core coincides with a strong concentration of magnetic field, which is not observed in this SGS. Both structures have a bright centre, although caused by different reasons: rosette brightening is due to a reduced opacity produced by the magnetic field, while in the SGS it is due to a velocity gradient which, in the $\mathrm{Ca}$ II $\mathrm{K}$ spectral line, blue-shifts the $K_{3}$ feature and enhances the $K_{2 \text { r }}$ peak.

The chromospheric velocity map (Fig. 7b) shows a clear flow towards the network boundaries but the interpretation of the Doppler shift in the SGS core is non-trivial. To explain the blue-shift observed in the chromospheric lines, especially in the $\mathrm{Ca}$ II $\mathrm{K}$, we propose two scenarios that take into account the magnetic field topology.

1. In the first scenario, depicted in Fig. 12a, we assume that the fibrils coinciding with the blue-shifts are totally vertical or slightly inclined towards the observer and, hence, the blue-shift is an actual upflow. Thus, the flow would be directed from the SGS centre towards the network. The SGS is not only the apparent convergence point for the fibrils, but also the location of flux emergence two days after the SST observation. We could therefore interpret the strong blue-shift as a hint of an exiguous undetected flux emergence taking place during the CRISP 

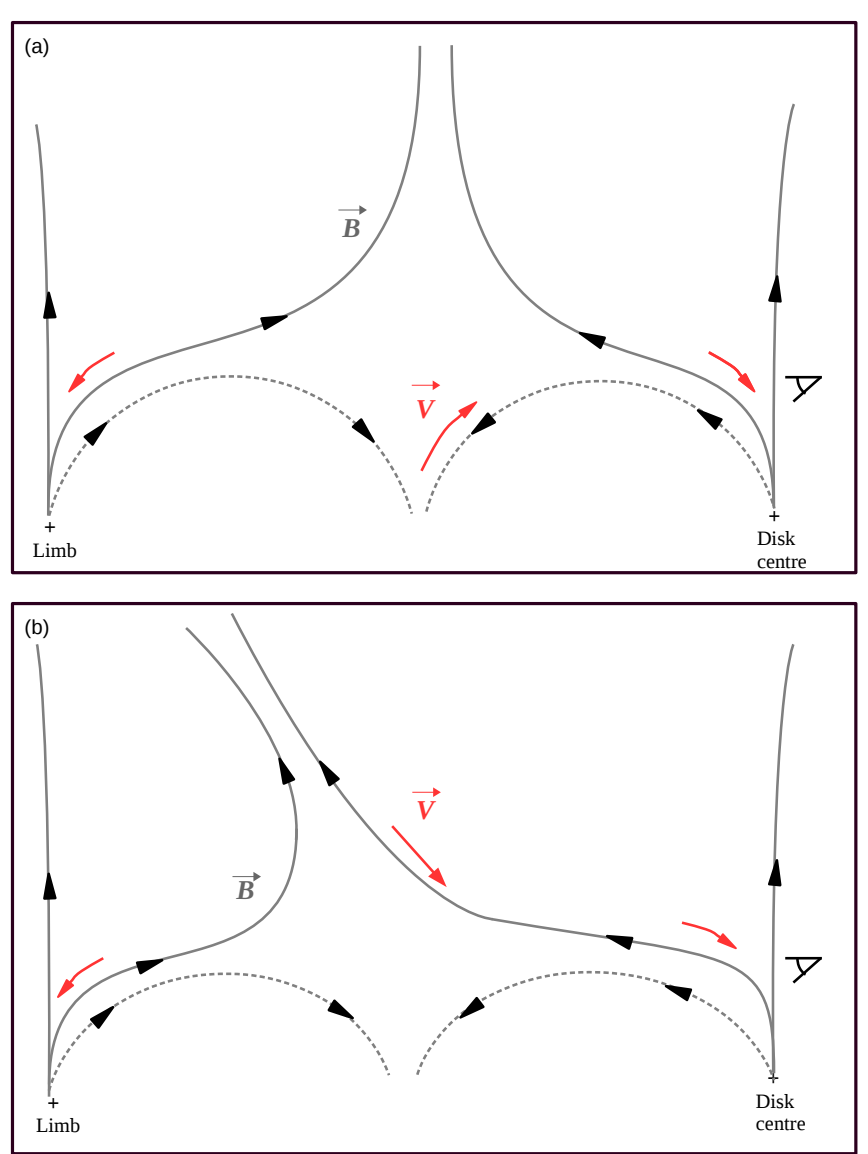

Fig. 12. Chromospheric magnetic field and velocity models above a section of the supergranular structure. The grey lines indicate the magnetic field lines and the red ones the velocity along the fibrils. Plus signs represent the positive field at the network boundaries. Panel $a$ : scenario 1 and panel $b$ : scenario 2 (see the text).

observations on 2017 April 20. However, we do not see anomalies in the photospheric LOS velocity or a distortion of the granules as expected in case of flux emergence (Cheung et al. 2007). 2. In the second scenario (Fig. 12b), we interpret the blue-shift as a downflow. We exclude the possibility of coronal material sliding down along a positive magnetic field canopy, since $\mathrm{H} \alpha$ does not show any trace of coronal rain. So, the downflow has to take place along fibrils inclined towards the limb. The different appearance of the fibrils on the east and west sides of the SGS could be a hint of such a magnetic field asymmetry. In that case, we would observe a draining inside loop-shaped fibrils, inclined according to the asymmetric canopy field. The draining flow is in agreement with the predominant bulk motion measured by Tsiropoula et al. (1993) in a typical rosette, although they also observe an upflow in its core. We believe that this second scenario is more likely since there is no clear indication of flux emergence in the photosphere.

\section{Summary}

We have retrieved the magnetic field and the LOS velocity field in the photosphere and chromosphere of a unipolar region of supergranular size. We summarise our conclusions as follows:

- The chromospheric magnetic field lines bend above the SGS suggesting a canopy-shaped configuration.
- The photospheric magnetic flux of the entire SGS is unbalanced.

- A strong velocity gradient along the LOS might be responsible for the bright SGS core.

- We interpret the $K_{3}$ blue-shift observed along the fibrils in the centre of the SGS as a draining flow.

Future studies should address the topology and velocity issues by observing SGSs on multiple days to avoid de-projection mistakes and uncertainties. Deep spectropolarimetry should be used to retrieve a more accurate inclination of the magnetic field in the chromosphere above SGS. Eventually, studying the time evolution will establish how long the canopy configuration remains coherent.

Acknowledgements. The Swedish 1-m Solar Telescope is operated by the Institute for Solar Physics of Stockholm University in the Spanish Observatorio del Roque de los Muchachos of the Instituto de Astrofísica de Canarias. We made use of resources provided by the Swedish National Infrastructure for Computing (SNIC) at the High Performance Computing Centre North at Umeå University. JdlCR is supported by grants from the Swedish Research Council (2015-03994), the Swedish National Space Board (128/15) and the Swedish Civil Contingencies Agency (MSB). This project has received funding from the European Research Council (ERC) under the European Union's Horizon 2020 research and innovation programme (SUNMAG, grant agreement 759548). This research was supported by the CHROMOBS and CHROMATIC grants of the Knut och Alice Wallenberg foundation. This research has made use of NASA's Astrophysical Data System.

\section{References}

Aiouaz, T., \& Rast, M. P. 2006, ApJ, 647, L183

Asensio Ramos, A., de la Cruz Rodríguez, J., Martínez González, M. J., \& Socas-Navarro, H. 2017, A\&A, 599, A133

Beckers, J. M. 1977, ApJ, 213, 900

Bjørgen, J. P., Sukhorukov, A. V., Leenaarts, J., et al. 2018, A\&A, 611, A62

Bommier, V. 2011, A\&A, 530, A51

Bommier, V., Martínez González, M., Bianda, M., et al. 2009, A\&A, 506, 1415 Cheung, M. C. M., Schüssler, M., \& Moreno-Insertis, F. 2007, A\&A, 467, 703

Dara-Papamargaritis, H., \& Koutchmy, S. 1983, A\&A, 125, 280

de la Cruz Rodríguez, J., \& Socas-Navarro, H. 2011, A\&A, 527, L8

de la Cruz Rodríguez, J., Kiselman, D., \& Carlsson, M. 2011, A\&A, 528, A113

de la Cruz Rodríguez, J., Löfdahl, M. G., Sütterlin, P., Hillberg, T., \& Rouppe van der Voort, L. 2015, A\&A, 573, A40

De Pontieu, B., Erdélyi, R., \& James, S. P. 2004, Nature, 430, 536

De Pontieu, B., McIntosh, S., Hansteen, V. H., et al. 2007, PASJ, 59, S655

Degl'Innocenti, M. L., \& Landolfi, M. 2004, Polarization in Spectral Lines (Springer Science \& Business Media), 307

Foukal, P. 1971, Sol. Phys., 19, 59

Gabriel, A. H. 1976, Phil. Trans. R. Soc. London, Ser. A, 281, 339

Gonzalez, R. C., \& Woods, R. E. 2002, Electron. Ind., 455

Gošić, M., Bellot Rubio, L. R., Orozco Suárez, D., Katsukawa, Y., \& del Toro Iniesta, J. C. 2014, ApJ, 797, 49

Hale, G. E., \& Ellerman, F. 1904, ApJ, 19, 41

Ishikawa, R., \& Tsuneta, S. 2011, ApJ, 735, 74

Jafarzadeh, S., Rutten, R. J., Solanki, S. K., et al. 2017, ApJS, 229, 11

Jefferies, S. M., McIntosh, S. W., Armstrong, J. D., et al. 2006, ApJ, 648, L151

Jones, H. P., \& Giovanelli, R. G. 1983, Sol. Phys., 87, 37

Kosugi, T., Matsuzaki, K., Sakao, T., et al. 2007, Sol. Phys., 243, 3

Lagg, A., Ishikawa, R., Merenda, L., et al. 2009, in The Second Hinode Science Meeting: Beyond Discovery-Toward Understanding, eds. B. Lites, M. Cheung, T. Magara, J. Mariska, \& K. Reeves, ASP Conf. Ser., 415, 327

Lagg, A., Lites, B., Harvey, J., Gosain, S., \& Centeno, R. 2017, Space Sci. Rev., 210,37

Langfellner, J., Gizon, L., \& Birch, A. C. 2015, A\&A, 579, L7

Leenaarts, J., Carlsson, M., \& Rouppe van der Voort, L. 2015, ApJ, 802, 136

Leka, K. D., Barnes, G., \& Crouch, A. 2014, Astrophysics Source Code Library [record ascl:1404.007]

Lemen, J. R., Title, A. M., Akin, D. J., et al. 2012, Sol. Phys., 275, 17

Lites, B. W., Kubo, M., Socas-Navarro, H., et al. 2008, ApJ, 672, 1237

Löfdahl, M. G., \& Scharmer, G. B. 1994, A\&AS, 107, 243

Löfdahl, M. G., Hillberg, T., de la Cruz Rodriguez, J., et al. 2018, ArXiv e-prints [arXiv:1804.03030]

Martínez-Sykora, J., De Pontieu, B., Carlsson, M., \& Hansteen, V. 2016, ApJ, 831, L1 
Metcalf, T. R. 1994, Sol. Phys., 155, 235

Mooroogen, K., Morton, R. J., \& Henriques, V. 2017, A\&A, 607, A46

Neckel, H. 1999, Sol. Phys., 184, 421

Orozco Suárez, D., \& Bellot Rubio, L. R. 2012, ApJ, 751, 2

Orozco Suárez, D., \& Del Toro Iniesta, J. C. 2007, A\&A, 462, 1137

Orozco Suárez, D., Bellot Rubio, L. R., \& Del Toro Iniesta, J. C. 2010, A\&A, 518, A3

Orozco Suárez, D., Katsukawa, Y., \& Bellot Rubio, L. R. 2012, ApJ, 758, L38

Pesnell, W. D., Thompson, B. J., \& Chamberlin, P. C. 2012, Sol. Phys., 275, 3

Pietarila, A., Hirzberger, J., Zakharov, V., \& Solanki, S. K. 2009, A\&A, 502, 647

Reardon, K. P., Wang, Y.-M., Muglach, K., \& Warren, H. P. 2011, ApJ, 742, 119

Rincon, F., Roudier, T., Schekochihin, A. A., \& Rieutord, M. 2017, A\&A, 599, A69

Rutten, R. J. 2006, in Solar MHD Theory and Observations: A High Spatial Resolution Perspective, eds. J. Leibacher, R. F. Stein, \& H. Uitenbroek, ASP Conf. Ser., 354, 276

Rutten, R. J. 2007, in The Physics of Chromospheric Plasmas, eds. P. Heinzel, I. Dorotovič, \& R. J. Rutten, ASP Conf. Ser., 368

Schad, T. A., Penn, M. J., \& Lin, H. 2013, ApJ, 768, 111

Scharmer, G. B. 2006, A\&A, 447, 1111
Scharmer, G. B., Bjelksjo, K., Korhonen, T. K., Lindberg, B., \& Petterson, B. 2003, in Innovative Telescopes and Instrumentation for Solar Astrophysics, eds. S. L. Keil, \& S. V. Avakyan, Proc. SPIE, 4853, 341

Scharmer, G. B., Narayan, G., Hillberg, T., et al. 2008, ApJ, 689, L69

Scherrer, P. H., Schou, J., Bush, R. I., et al. 2012, Sol. Phys., 275, 207

Schrijver, C. J., \& Title, A. M. 2003, ApJ, 597, L165

Schrijver, C. J., Cote, J., Zwaan, C., \& Saar, S. H. 1989, ApJ, 337, 964

Shine, R. A., Milkey, R. W., \& Mihalas, D. 1975, ApJ, 199, 724

Skumanich, A., \& Lites, B. W. 1987, ApJ, 322, 473

Solanki, S. K., \& Steiner, O. 1990, A\&A, 234, 519

Tian, H., Potts, H. E., Marsch, E., Attie, R., \& He, J.-S. 2010, A\&A, 519, A58 Tsiropoula, G., Alissandrakis, C. E., \& Schmieder, B. 1993, A\&A, 271, 574

Tsuneta, S., Ichimoto, K., Katsukawa, Y., et al. 2008, Sol. Phys., 249, 167

Tziotziou, K., Tsiropoula, G., \& Mein, P. 2003, A\&A, 402, 361

van Noort, M., Rouppe van der Voort, L., \& Löfdahl, M. G. 2005, Sol. Phys., 228,191

Wedemeyer-Böhm, S., Lagg, A., \& Nordlund, Å. 2009, Space Sci. Rev., 144, 317

Zhu, X., Wang, H., Du, Z., \& He, H. 2016, ApJ, 826, 51

Zhugzhda, I. D., \& Dzhalilov, N. S. 1984, A\&A, 132, 45 